\title{
How usual is usual care in pragmatic intervention studies in primary care? An overview of recent trials
}

\author{
Antonia FH Smelt, Gerda M van der Weele, Jeanet W Blom, Jacobijn Gussekloo \\ and Willem JJ Assendelft
}

\begin{abstract}
Background

Because pragmatic trials are performed to determine if an intervention can improve current practice, they often have a control group receiving 'usual care'. The behaviour of caregivers and patients in this control group should be influenced by the actions of researchers as little as possible. Guidelines for describing the composition and management of a usual care control group are lacking.

Aim

To explore the variety of approaches to the usual care concept in pragmatic trials, and evaluate the influence of the study design on the behaviour of caregivers and patients in a usual care control group.

Design of study

Review of 73 pragmatic trials in primary care with a usual care control group published between January 2005 and December 2009 in the British Medical Journal, the British Journal of General Practice, and Family Practice. Outcome measures were: description of the factors influencing caregiver and patients in a usual care control group related to an individual randomised design versus cluster randomisation. Results

In total, 38 individually randomised trials and 35 cluster randomised trials were included. In most trials, caregivers had the freedom to treat control patients according to their own insight; in two studies, treatment options were restricted. Although possible influences on the behaviour of control caregivers and control patients were more often identified in individually randomised trials, these influences were also present in cluster randomised trials. The description of instructions and information provided to the control group was often insufficient, which made evaluation of the trials difficult.

Conclusion

Researchers in primary care medicine should carefully consider the design of a usual care control group, especially with regard to minimising the risk of studyinduced behavioural change. It is recommended that an adequate description of the information is provided to control caregivers and control patients. A proposal is made for an extension to the CONSORT statement that requires authors to specify details of the usual care control group.

Keywords

control groups; family practice; pragmatic trials; primary care; usual care.
\end{abstract}

\section{INTRODUCTION}

Many trials in primary care require a pragmatic design. In contrast to explanatory trials, which are performed under ideal and controlled conditions, pragmatic trials measure the effect of an intervention in real clinical practice. Because pragmatic trials are performed to determine whether the intervention can improve current practice, they often have a 'usual care' control group. The care received by this control group is supposed to reflect the care as usually received by patients in daily practice.

The design of a trial with a usual care control group requires specific attention (Box 1). The main difficulty is to ensure that this control group receives genuine usual care as supplied in everyday practice. ${ }^{2}$ However, various actions by the researchers may influence the behaviour of caregivers and patients. For example, behavioural change of control caregivers may be induced when they are informed about the issues under study, or because of a learning effect when they have to provide usual care to one patient and an intervention to another. Behavioural change of control patients may be induced when they are briefed about the trial and asked to give informed consent, or when they are

AFH Smelt, MD, junior researcher, GP trainee; GM van der Weele, GP, junior researcher; JW Blom, MD, PhD, senior researcher; J Gussekloo, MD, professor of general practice; WJJ Assendelft, MD, professor of general practice, Department of Public Health and Primary Care, Leiden University Medical Center, Leiden, the Netherlands.

Address for correspondence

Ms Antonia Smelt, Leiden University Medical Center, Public Health and Primary Care, Postbox 9600, Postzone V-0-P, Leiden, 2300 RC, Netherlands. E-mail: a.f.h.smelt@lumc.nl

Submitted: 26 June 2009; Editor's response: 27 August 2009; final acceptance: 22 January 2010.

() British Journal of General Practice

This is the full-length article of an abridged version published in print. Cite this article as: Br J Gen Pract 2010; DOI: 10.3399/bjgp10X514819. 
asked to complete questionnaires and undergo examinations, drawing their attention to their condition and possible interventions. These actions may change their help-seeking behaviour or influence their complaints. This, in turn, may affect the outcome and the interpretation of the trial. Consequently, researchers have to think carefully about the influence of the study information and the intervention on the control group and on how to minimise this effect.

Researchers have different opinions about the meaning of the term 'usual care' and, consequently, apply different methods when designing and reporting a trial with a usual care control group. Guidelines on the reporting of trials are available to researchers in the CONSORT statement, including its extension to cluster randomised trials, and its recently published extension to pragmatic trials. ${ }^{3-5}$ However, these guidelines do not cover the specific requirements for describing the composition and management of a usual care control group. Two recent reviews on the validity of pragmatic cluster randomised trials focused on other issues, that is, mainly on the risk of selection bias. Until now, the risk of behavioural change of caregivers and patients in trials with a usual care group has been discussed only marginally. ${ }^{6,7} \mathrm{~A}$ recent review on low back pain reported a poor description of the usual care control arm in 26 of 33 reviewed studies. ${ }^{8,9}$

To gain insight into the variety of approaches to the usual care concept in primary care research, this study explored a cohort of pragmatic trials with a usual care control group, reporting for individually randomised and cluster-randomised studies separately. Furthermore, it examined the possible influences of the study information and awareness of the intervention on the behaviour of control caregivers and control patients. This overview shows the problems researchers face when designing pragmatic trials with a usual care control group.

\section{METHOD}

One of the authors manually searched three medical journals: the British Medical Journal, the British Journal of General Practice, and Family Practice, from January 2005 to December 2009. These particular journals were selected because they regularly publish articles on pragmatic trials in primary care; thus, this overview was not intended to be exhaustive. The two criteria for inclusion were:

- A randomised pragmatic or effectiveness trial in a primary care or nursing home population; the aim of the trial should be to evaluate the overall effectiveness of an intervention in a 'real-life' situation, when people may not receive all of the treatment, and may use other treatments as well,

\section{How this fits in}

For pragmatic trials researchers often use a usual care control group. However, this concept is defined in a variety of ways. In addition, the information and treatment provided to the control group is often scarcely described. This makes it difficult for readers of trial articles to assess the applicability of trials with a usual care control group to their own population. This review proposes a more detailed description of the control group in CONSORT statement as a first step to the solution of this problem.

\section{Box 1. Example of dilemmas faced by the present authors when designing a pragmatic trial with a usual care control group.}

Guidelines give clear advice about prophylactic medication for migraine patients with frequent headaches. However, GPs do not often prescribe this medication. This study's authors decided to perform a randomised clinical trial to establish the advantages of a new intervention aimed at improving migraine treatment by GPs. Most likely this will be a pragmatic trial with one group of GPs applying the intervention and another group continuing their normal way of working ('usual care'). For an optimal comparison it is preferable if control patients and control physicians are unaware of the study. However, patients have to be invited to participate, need to provide informed consent, and have to complete questionnaires to measure the outcome. Furthermore, control physicians have to be informed and agree to participate. Therefore, physicians and patients will be aware of the study and this could change or adapt their normal behaviour. For example, physicians could start studying the available guidelines on migraine and subsequently re-evaluate the therapy options for their migraine patients; patients might decide to visit their physician after reading the information and filling in the questionnaire; and, just after the study has started, an update of the national headache guideline might be sent to all GPs. For both the researcher and the reader it is difficult to estimate the impact of all these possible influences. the trial has broad eligibility criteria, and patient and practice-oriented outcomes. ${ }^{10,11}$
Figure 1. Flowchart of the search and results.

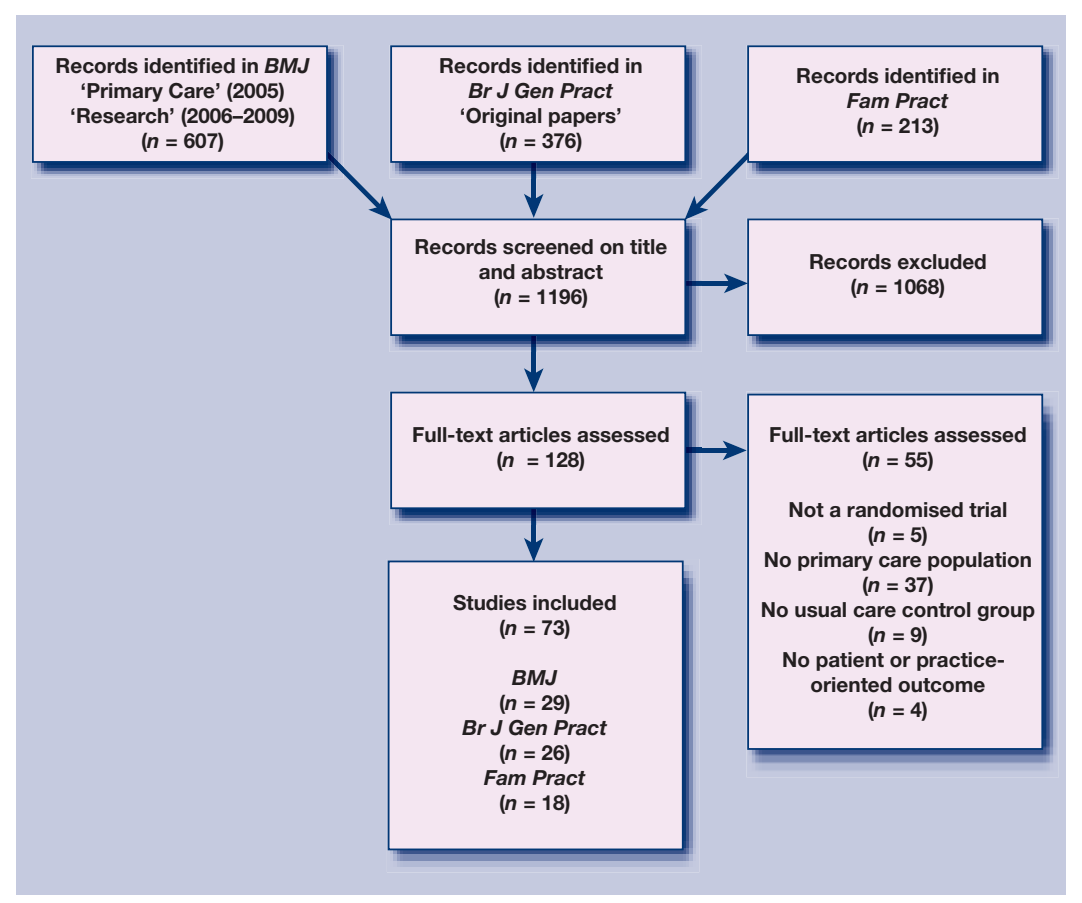


Table 1. Description of individually randomised trials $(n=38)$ as reported by the authors of the trials.

First author, year of publication

Boivin, 2008 ${ }^{12}$ Study subject

Multifactorial strategy of pain management during vaccination to decrease pain

Community Pharmacy Community pharmacy-led medicines Medicines Management management for patients with $\mathrm{CHD}$ in primary Project Evaluation Team, care to improve secondary prevention $2007^{13}$

\begin{tabular}{|c|c|c|c|}
\hline Christensen, $2005^{14}$ & $\begin{array}{l}\text { A composite } S Q \text { to enhance } \\
\text { recognition and treatment of functional } \\
\text { illness in primary care in consecutive } \\
\text { patients presenting with a new health problem }\end{array}$ & $\begin{array}{l}\text { Patients complete SQ, content } \\
\text { disclosed to GP }\end{array}$ & $\begin{array}{l}\text { Patients complete SQ, no } \\
\text { disclosure to GP }\end{array}$ \\
\hline Coppin, $2008^{15}$ & $\begin{array}{l}\text { Managing ear wax in } \\
\text { primary care }\end{array}$ & $\begin{array}{l}\text { Self-treatment with eardrops } \\
\text { and bulb syringe }\end{array}$ & $\begin{array}{l}\text { Routine care (ear drops for } \\
2 \text { days, then irrigation in practice) }\end{array}$ \\
\hline Crilly, $2005^{16}$ & $\begin{array}{l}\text { Provision of an educational booklet in adults } \\
\text { prescribed thyroxine for primary hypothyroidism } \\
\text { to improve adherence in primary care }\end{array}$ & $\begin{array}{l}\text { Educational booklet addressing lay } \\
\text { health beliefs about medicine taking }\end{array}$ & Usual care \\
\hline Daley, $2008^{17}$ & $\begin{array}{l}\text { Feasibility of an exercise intervention for } \\
\text { women with postnatal depression }\end{array}$ & $\begin{array}{l}\text { Two one-to-one exercise consultations } \\
\text { by researcher }\end{array}$ & $\begin{array}{l}\text { Usual care, consultation at end } \\
\text { of study }\end{array}$ \\
\hline Dennis, $2009^{18}$ & $\begin{array}{l}\text { Effect of peer support on prevention of } \\
\text { postnatal depression among high-risk women }\end{array}$ & No peer support & $\begin{array}{l}\text { Telephone-based peer (mother-to } \\
\text {-mother) support }\end{array}$ \\
\hline Farmer, $2007^{19}$ & $\begin{array}{l}\text { Impact of self-monitoring of blood glucose } \\
\text { in the management of patients with } \\
\text { non-insulin-treated diabetes }\end{array}$ & $\begin{array}{l}\text { Usual care }+ \text { blood glucose } \\
\text { self-monitoring }\end{array}$ & $\begin{array}{l}\text { Standardised usual care } \\
\text { (3-monthly measurement } \\
\text { of } \mathrm{Hb}_{1 \mathrm{c}} \text { ) }\end{array}$ \\
\hline Gorgels, $2007^{20}$ & $\begin{array}{l}\text { Reducing psychotropic medication prescription } \\
\text { in long-term benzodiazepine users }\end{array}$ & $\begin{array}{l}\text { Discontinuation letter + taper scheme } \\
\text { with/without group psychotherapy }\end{array}$ & $\begin{array}{l}\text { Discontinuation letter + } \\
\text { usual care }\end{array}$ \\
\hline Green, $2007^{21}$ & Treatment of menopausal symptoms & Treatment by qualified herbal practitioner & Waiting list \\
\hline Griffiths, $2005^{22}$ & $\begin{array}{l}\text { Improving self-efficacy in Bangladeshi } \\
\text { patients with chronic disease in primary care }\end{array}$ & $\begin{array}{l}\text { Self-management programme provided } \\
\text { by Bangladeshi lay tutors }\end{array}$ & Waiting list \\
\hline Gruffydd-Jones, $2005^{23}$ & $\begin{array}{l}\text { The effectiveness of targeted asthma care } \\
\text { in general practice using telephone triage }\end{array}$ & 6-monthly check-up by telephone & $\begin{array}{l}\text { Usual care by } 6 \text {-monthly } \\
\text { check-up via an appointment } \\
\text { with asthma nurse }\end{array}$ \\
\hline Hamilton, $2007^{24}$ & $\begin{array}{l}\text { The effect of a patient SCAF on prescribing, } \\
\text { adherence and patient satisfaction }\end{array}$ & $\begin{array}{l}\text { Patient completes SCAF in waiting room, } \\
\text { SCAF given to GP on entry to } \\
\text { consultation room }\end{array}$ & No SCAF \\
\hline Hoefman, $2005^{25}$ & $\begin{array}{l}\text { Feasibility of patient-activated loop records } \\
\text { for detecting heart rhythm abnormalities } \\
\text { in patients with new episodes of } \\
\text { palpitations or light-headedness and } \\
\text { normal ECG primary care }\end{array}$ & $\begin{array}{l}\text { Loop recorder for a maximum } \\
\text { of } 4 \text { weeks + usual care from GP }\end{array}$ & $\begin{array}{l}\text { Usual care (patients included } \\
\text { when routine ECG showed no } \\
\text { abnormalities) }\end{array}$ \\
\hline Holland, $2007^{26}$ & $\begin{array}{l}\text { Visits from community pharmacists for patients } \\
\text { diagnosed with heart failure after an } \\
\text { emergency admission to reduce hospital } \\
\text { readmissions }\end{array}$ & $\begin{array}{l}\text { Pharmacists provided with copy } \\
\text { of discharge letter, home visit by } \\
\text { pharmacist within } 2 \text { weeks } \\
\text { of discharge }\end{array}$ & $\begin{array}{l}\text { Usual care by GP and } \\
\text { community pharmacist }\end{array}$ \\
\hline Holland, $2005^{27}$ & $\begin{array}{l}\text { Home-based medication review by local } \\
\text { pharmacist in older people discharged from } \\
\text { hospital to reduce emergency admissions }\end{array}$ & $\begin{array}{l}\text { Pharmacist provided with copy of } \\
\text { discharge letter, home-based } \\
\text { medication review by pharmacist }\end{array}$ & $\begin{array}{l}\text { Usual care by GP and } \\
\text { community pharmacist }\end{array}$ \\
\hline Hunkeler, $2006^{28}$ & $\begin{array}{l}\text { Collaborative care intervention for } \\
\text { depressed older in primary care }\end{array}$ & $\begin{array}{l}\text { Proactive depression treatment } \\
\text { by depression care manager (nurse), } \\
\text { GP, psychiatrist, and liaison GP }\end{array}$ & Usual care \\
\hline Khunti, $2006^{29}$ & $\begin{array}{l}\text { Effect of near-patient testing for } \mathrm{HbA}_{1 c} \text { in } \\
\text { people with type } 2 \text { diabetes mellitus on } \\
\text { glycaemic control in primary care }\end{array}$ & $\begin{array}{l}\text { Rapid test for } \mathrm{HbA}_{1 c} \text { (practices } \\
\text { also continued usual follow-up) }\end{array}$ & $\begin{array}{l}\text { Routine care (laboratory testing } \\
\text { for } \mathrm{Hb} A_{1 c} \text { ) }\end{array}$ \\
\hline Lawton, $2009^{30}$ & $\begin{array}{l}\text { Effectiveness of a programme of exercise } \\
\text { on prescription among relatively inactive } \\
\text { women on physical activity }\end{array}$ & $\begin{array}{l}\text { Brief physical activity interventionby nurse } \\
\text { with } 6 \text {-month follow-up visit and monthly } \\
\text { telephone support over } 9 \text { months }\end{array}$ & Usual care \\
\hline
\end{tabular}

Description of care in control group

Multifactorial strategy including Usual care

pharmacological and non-pharmacological approaches

Consultation of $\mathrm{CHD}$ patients by community pharmacist;

Usual care from GP and community pharmacist recommendations sent to GP
Patients complete SQ, content

Self-treatment with eardrops

bulb syring

ducational booklet addressing lay

Two one-to-one exercise consultations by researcher

Usual care + blood glucose

Discontinuation letter + taper schem

Usual care by 6-monthly check via an appointment with asthma nurse

SCAF given to GP on entry to

Loop recorder for a maximum hen routine ECG showed no for detecting heart rhythm abnormalities palpitations or light-headedness and G primary care diagnosed with heart failure after an readmission pharmacist in older people discharged from

Collaborative care intervention for telephone support over 9 months 
Table 1 continued. Description of individually randomised trials $(n=38)$ as reported by the authors of the trials. Leong, $2006^{31} \quad \begin{aligned} & \text { The use of text messaging to improve } \\ & \text { attendance in primary care }\end{aligned}$
Reminder via text message or mobile phone call $24-48$ hours prior to appointment

Liew, 200932

Text messaging reminders to reduce non-

Text message or telephone reminder attendance in chronic disease follow-up

\begin{tabular}{ll}
\hline Linschoten, 2009 & $\begin{array}{l}\text { Supervised exercise therapy for } \\
\text { patellofemoral pain syndrome }\end{array}$ \\
\hline Little, 2008 & Alexander technique lessons, exercise, and \\
& massage for chronic and recurrent back pain \\
\hline Martins, 200935 & $\begin{array}{l}\text { Food incentives to improve completion of } \\
\text { tuberculosis treatment }\end{array}$ \\
\hline McMahon, 2007 & $\begin{array}{l}\text { Graduate mental health worker case } \\
\text { management of depression in people using } \\
\text { antidepressants for more than 2 months }\end{array}$ \\
\hline McManus, 2005 & $\begin{array}{l}\text { Improving blood pressure control in primary } \\
\text { care by self-monitoring }\end{array}$
\end{tabular}

Muirhead, $2006^{38}$

Effect of organised and supervised peer support on initiation and duration of breastfeeding

No reminder

No reminder

Standardised exercise programme

Usual care ('wait and see' approach)

Massage/Alexander technique lessons/ Normal care by GP exercise

Nutritious, culturally appropriate daily Routine care (nutritional advice) meal and food package

Usual care + case management from Usual care, all prescribed graduate primary care mental health antidepressant worker

Monthly blood pressure

measurement by patient, patient card with target blood pressure

Usual care (blood pressure monitoring by GP, information sheet on self-help measures)

Normal breastfeeding support (community midwife first 10 days, thereafter health visitor, breastfeeding support groups and breastfeeding workshops)

\begin{tabular}{|c|c|c|c|}
\hline Nanchahal, $2009^{39}$ & $\begin{array}{l}\text { Weight-management intervention for adults } \\
\text { with body mass index } \geq 27 \mathrm{~kg} / \mathrm{m}^{2}\end{array}$ & $\begin{array}{l}\text { Nurse-led weight- management } \\
\text { programme }\end{array}$ & Usual care \\
\hline Norg, $2006^{40}$ & $\begin{array}{l}\text { Treatment protocol for male lower urinary } \\
\text { tract symptoms to reduce symptoms }\end{array}$ & $\begin{array}{l}\text { Comprehensive treatment } \\
\text { protocol by researcher }\end{array}$ & Usual care by GP \\
\hline Roberts, $2006^{41}$ & $\begin{array}{l}\text { Effectiveness of hypnotherapy as a } \\
\text { complementary therapy in the primary care } \\
\text { management of irritable bowel syndrome }\end{array}$ & $\begin{array}{l}\text { Usual practice + five sessions of } \\
\text { hypnotherapy }\end{array}$ & Usual practice \\
\hline Schreuders, $2005^{42}$ & $\begin{array}{l}\text { Effect of problem-solving treatment for patients } \\
\text { with mental health problems on feelings of } \\
\text { depression and anxiety, and on attendance rates }\end{array}$ & $\begin{array}{l}\text { Problem-solving treatment by } \\
\text { mental health nurse }\end{array}$ & Usual care by GP \\
\hline Schroeder, $2005^{43}$ & $\begin{array}{l}\text { Effectiveness of nurse-led adherence support } \\
\text { in hypertensive patients }\end{array}$ & $\begin{array}{l}\text { Usual care }+ \text { blood pressure checks and } \\
\text { adherence support sessions by } \\
\text { practice nurse }\end{array}$ & $\begin{array}{l}\text { Usual care }+ \text { blood pressure } \\
\text { checks at similar intervals as } \\
\text { intervention group }\end{array}$ \\
\hline Thomas, $2006^{44}$ & $\begin{array}{l}\text { Acupuncture for persistent non-specific } \\
\text { low back pain }\end{array}$ & Short course of traditional acupuncture & $\begin{array}{l}\text { Usual care (NHS treatment } \\
\text { according to GP's assessment } \\
\text { of needs) }\end{array}$ \\
\hline Thomsen, $2005^{45}$ & $\begin{array}{l}\text { Effect of preventive health screening and health } \\
\text { discussions on primary care utilisation in } \\
\text { primary care }\end{array}$ & $\begin{array}{l}\text { (1) health screening, or } \\
\text { (2) health discussion }\end{array}$ & $\begin{array}{l}\text { No invitation for screening } \\
\text { or discussion }\end{array}$ \\
\hline van Rijn, $2007^{46}$ & $\begin{array}{l}\text { Supervised exercises for adults with acute } \\
\text { lateral ankle sprain }\end{array}$ & $\begin{array}{l}\text { Conventional treatment }+ \text { supervised } \\
\text { exercises }\end{array}$ & $\begin{array}{l}\text { Conventional treatment } \\
\text { (information about mobilisation } \\
\text { and home exercises) }\end{array}$ \\
\hline Vicens, $2006^{47}$ & $\begin{array}{l}\text { Structured intervention aimed at withdrawal } \\
\text { from long-term benzodiazepine use }\end{array}$ & $\begin{array}{l}\text { Standardised interview + stepwise } \\
\text { dose reduction by GP }\end{array}$ & $\begin{array}{l}\text { Usual care by GP, after being } \\
\text { informed of convenience of } \\
\text { reducing benzodiazepine use }\end{array}$ \\
\hline Wake, $2009^{48}$ & Intervention for overweight or obese children & $\begin{array}{l}\text { Four standard consultations targeting } \\
\text { change in nutrition, physical activity, and } \\
\text { sedentary behaviour }\end{array}$ & No consultations \\
\hline Williams, $2005^{49}$ & $\begin{array}{l}\text { Effectiveness of a nurse-led continence } \\
\text { service for individuals reporting } \\
\text { urinary symptoms }\end{array}$ & $\begin{array}{l}\text { Continence service by specially } \\
\text { trained nurse }\end{array}$ & $\begin{array}{l}\text { Standard care, individuals } \\
\text { provided with leaflet detailing how } \\
\text { to access existing continence } \\
\text { services or GP }\end{array}$ \\
\hline
\end{tabular}

$C H D=$ coronary heart disease. $E C G=$ electrocardiogram. $S C A F=$ self-completed agend form. $S Q=$ screening questionnaire. 
Table 2. Evaluation of the individually randomised trials $(n=38)$.

\begin{tabular}{|c|c|c|c|c|c|c|c|c|c|c|c|}
\hline \multirow[b]{2}{*}{$\begin{array}{l}\text { In } \\
\text { allc } \\
\mathrm{p}\end{array}$} & \multicolumn{5}{|c|}{ Influence on caregivers } & \multicolumn{6}{|c|}{ Influence on control patients } \\
\hline & $\begin{array}{l}\text { Informed } \\
\text { about } \\
\text { allocation of } \\
\text { patients }\end{array}$ & $\begin{array}{l}\text { Informed } \\
\text { about Q } \\
\text { content of } \\
\text { intervention }\end{array}$ & $\begin{array}{c}\text { Questionnaires } \\
\text { before or } \\
\text { during trial }\end{array}$ & $\begin{array}{l}\text { Extra } \\
\text { information/ } \\
\text { training on } \\
\text { the subject }\end{array}$ & $\begin{array}{c}\text { Risk of } \\
\text { learning } \\
\text { effect } \\
\text { by treating } \\
\text { intervention } \\
\text { patients }\end{array}$ & $\begin{array}{l}\text { Informed } \\
\text { consent } \\
\text { given }\end{array}$ & $\begin{array}{l}\text { Informed } \\
\text { about } \\
\text { content of } \\
\text { intervention }\end{array}$ & $\begin{array}{c}\text { Provided } \\
\text { with } \\
\text { extra } \\
\text { information }\end{array}$ & $\begin{array}{l}\text { Informed } \\
\text { about } \\
\text { allocation }\end{array}$ & $\begin{array}{l}\text { Questionnaires/ } \\
\text { examinations }\end{array}$ & $\begin{array}{c}\text { Risk of } \\
\text { contact } \\
\text { with } \\
\text { intervention } \\
\text { patients }\end{array}$ \\
\hline Boivin, $2008^{12}$ & + & + & + & + & - & _ & $?$ & - & + & + & - \\
\hline Christensen, $2005^{14}$ & + & + & + & + & + & + & $?$ & $?$ & + & + & - \\
\hline Coppin, $2008^{15}$ & + & + & - & - & - & + & $?$ & $?$ & $?$ & + & - \\
\hline Crilly, $2005^{16}$ & - & - & - & - & - & + & - & - & + & + & - \\
\hline Dennis, $2009^{18}$ & - & $?$ & - & $?$ & - & + & $?$ & $?$ & + & + & $?$ \\
\hline Farmer, $2007^{19}$ & $?$ & $?$ & - & $?$ & + & + & $?$ & + & $?$ & + & - \\
\hline Gorgels, $2007^{20}$ & $?$ & + & - & + & + & + & $?$ & $?$ & + & - & - \\
\hline Green, $2007^{21}$ & $?$ & $?$ & - & $?$ & - & + & $?$ & $?$ & $?$ & + & - \\
\hline Griffiths, $2005^{22}$ & $?$ & $?$ & - & - & - & + & $?$ & $?$ & $?$ & + & - \\
\hline Gruffydd-Jones, $2005^{23}$ & $5^{23}+$ & + & - & + & + & + & $?$ & $?$ & + & + & - \\
\hline Hamilton, $2007^{24}$ & + & + & - & - & + & + & $?$ & - & $?$ & + & + \\
\hline Hoefman, $2005^{25}$ & + & + & + & + & + & + & $?$ & + & $?$ & + & - \\
\hline Holland, $2007^{26}$ & $?$ & $?$ & - & - & $?$ & + & $?$ & $?$ & + & + & - \\
\hline Holland, $2005^{27}$ & - & $?$ & - & $?$ & + & + & $?$ & $?$ & + & + & - \\
\hline Hunkeler, $2006^{28}$ & + & $?$ & - & + & + & + & $?$ & + & + & + & - \\
\hline Khunti, 2006 29 & + & + & - & - & - & + & + & - & + & + & - \\
\hline Lawton, 200930 & $?$ & $?$ & - & $?$ & $?$ & + & $?$ & - & $?$ & + & $?$ \\
\hline Linschoten, $2009^{33}$ & $?$ & $?$ & - & $?$ & $?$ & + & $?$ & + & + & + & - \\
\hline Little, $2008^{34}$ & - & $?$ & - & - & - & + & + & - & + & + & - \\
\hline Martins, $2009^{35}$ & + & + & - & $?$ & - & $?$ & $?$ & - & + & + & - \\
\hline McMahon, $2007^{36}$ & $?$ & $?$ & - & $?$ & + & + & $?$ & $?$ & $?$ & + & - \\
\hline McManus, $2005^{37}$ & + & + & - & - & + & + & $?$ & + & + & + & + \\
\hline Muirhead, $2006^{38}$ & $?$ & $?$ & - & - & - & + & $?$ & - & + & + & - \\
\hline Nanchahal, 2009 & $?$ & $?$ & - & $?$ & - & + & $?$ & - & $?$ & + & - \\
\hline Norg, $2006^{40}$ & + & - & - & - & - & + & - & - & + & + & - \\
\hline Roberts, $2006^{41}$ & + & + & - & + & - & + & $?$ & + & + & + & - \\
\hline Schreuders, $2005^{42}$ & $?$ & $?$ & - & $?$ & $?$ & + & $?$ & $?$ & $?$ & + & - \\
\hline Schroeder, $2005^{43}$ & + & + & - & + & + & + & $?$ & $?$ & + & + & - \\
\hline Thomas, $2006^{44}$ & + & + & - & - & - & + & $?$ & $?$ & + & + & - \\
\hline Thomsen, $2005^{45}$ & $?$ & + & - & + & + & - & - & - & - & - & - \\
\hline van Rijn, $2007^{46}$ & - & $?$ & - & $?$ & - & + & $?$ & - & $?$ & + & - \\
\hline Vicens, $2006^{47}$ & $?$ & + & - & + & + & + & $?$ & + & $?$ & + & - \\
\hline Wake, $2009^{48}$ & + & + & - & + & + & + & $?$ & $?$ & + & + & $?$ \\
\hline Williams, $2005^{49}$ & $?$ & $?$ & - & $?$ & - & + & + & + & + & + & - \\
\hline
\end{tabular}

$+=$ risk present; $-=$ risk not present; $?=$ not described in the article.

- The control group was reported to receive usual care; in case the term 'usual care' was not explicitly used, the paper reported on a comparison between the intervention(s) under study and normal practice (that is, it did not compare two different unrelated interventions). 
Table 3. Description of cluster randomised trials $(n=35)$ as reported by the authors of trials.

First author,

year of publication

Bebb, $2007^{50}$

\author{
Study subject
}

A treatment algorithm for hypertension in

patients with type 2 diabetes

Bellon, $2008^{51}$

Effectiveness of a GP intervention to reduce

frequent-attender consultations for frequent attendance

Cals, 200952 Intervention to reduce antibiotic use in lower respiratory tract infections

Cullen, 2006 $\quad$ Intervention to support the implementation of clinical guidelines for hepatitis management among current or former drug users
Intervention

Treatment by GP and practice nurse according to algorithm for treatment and monitoring of hypertension

GP training session on '7 hypotheses + team' intervention aimed at discovering reasons

\section{Reactive protein testing and/or training Usual care}

in enhanced communication skills

Educational sessions for GP on new Usual care by GP

guidelines, implementation, and nursing support in control group

Usual care

usual care
Description of care

No training GP, GP provides

\section{Structured group education programme Usual care}

in the community

with newly diagnosed type 2 diabetes de Groot, 200755 Cognitive behavioural therapy to prevent complicated grief among relatives and spouses bereaved by suicide

Downs, 2006 ${ }^{56} \quad$ Educational interventions to improve detection and management of dementia in primary care

Fitzmaurice, $2007^{57}$

Detection of atrial fibrillation in patients aged $\geq 65$ years

Francis, $2009^{58}$

Effect of booklet in primary care consultations on reconsulting and antibiotic prescribing

Harmsen, $2005^{59}$

Effect of an education intervention on intercultural communication between GP and patients of mutual understanding and quality of life

\begin{tabular}{|c|c|}
\hline Hayward, $2006^{60}$ & $\begin{array}{l}\text { Influenza vaccine programme for care home } \\
\text { staff to prevent death, morbidity, and health } \\
\text { service use among residents }\end{array}$ \\
\hline Hoddinott, $2009^{61}$ & $\begin{array}{l}\text { Effectiveness of policy to provide breastfeeding } \\
\text { groups for pregnant and breastfeeding } \\
\text { mothers on breastfeeding rates }\end{array}$ \\
\hline
\end{tabular}

\begin{tabular}{ll}
\hline Hogg, $2008^{62}$ & $\begin{array}{l}\text { A comprehensive preventive intervention } \\
\text { programme to improve preventive } \\
\text { care delivery }\end{array}$ \\
\hline
\end{tabular}

Janssen, 200963 Intensive multifactorial treatment for cardiovascular risk in patients with type 2 diabetes

Jellema, 2005 $\quad$ Treatment of low back pain aimed at psychosocial prognostic factors

Kerse, 2008 $\quad$ Effectiveness of an activity programme in improving function, quality of life, and falls in older people in residential care
Grief counselling programme by
Care as usual psychiatric nurse

(1) Electronic tutorial for GP, or

(2) decision support software, or (3)

workshop on appropriate treatment

Systematic screening ECG or opportunistic screening (pulse taking taking and ECG if pulse irregular)

Booklet on respiratory tract infections in Usual care children used during consultation and provided as a take-home resource
Videotape instruction for patients
No intervention

Only visited to collect data and training for GP

Primary healthcare team receives no education

Staff influenza vaccination promoted Usual policy; not actively by lead nurses promoting staff vaccination

No new breastfeeding groups

New breastfeeding groups, provide population coverage

\section{Monthly visit of practice by prevention No facilitator visits}

facilitator delivering an intervention strategy aimed at improving preventive care

Intensive treatment of glucose, blood Routine care according to 1999 pressure, and lipids, and structured guidelines from the Dutch lifestyle education

Minimal intervention strategy aimed at psychosocial prognostic factors by GP

Goal setting and activities of daily living Social visits by social programme by visiting gerontology nurse gerontologist who discussed and expert with a physiotherapist

\begin{tabular}{llll}
\hline Lester, 200766 & $\begin{array}{l}\text { Effectiveness of primary care mental } \\
\text { workers in improving patient satisfaction }\end{array}$ & Access to mental health worker & $\begin{array}{l}\text { No access to mental health } \\
\text { worker }\end{array}$ \\
\hline Lester, 200967 & $\begin{array}{l}\text { Effect of GP training in first-episode psychosis } \\
\text { on referral rates and duration of untreated } \\
\text { psychosis }\end{array}$ & $\begin{array}{l}\text { Educational intervention for GPs on } \\
\text { important symptoms and signs, } \\
\text { questioning skills, positive attitudes }\end{array}$ & No intervention for GPs \\
\hline Lo Fo, 2006 & $\begin{array}{l}\text { Increasing awareness of } \\
\text { intimate partner abuse }\end{array}$ & $\begin{array}{l}\text { (1) Full training; focus group and training } \\
\text { session on partner abuse, or (2) focus group }\end{array}$ \\
\hline
\end{tabular}
documented social activities and networks College of General Practitioners

Usual care by $G P$ 
Table 3 continued. Description of cluster randomised trials $(n=35)$ as reported by the authors of trials.

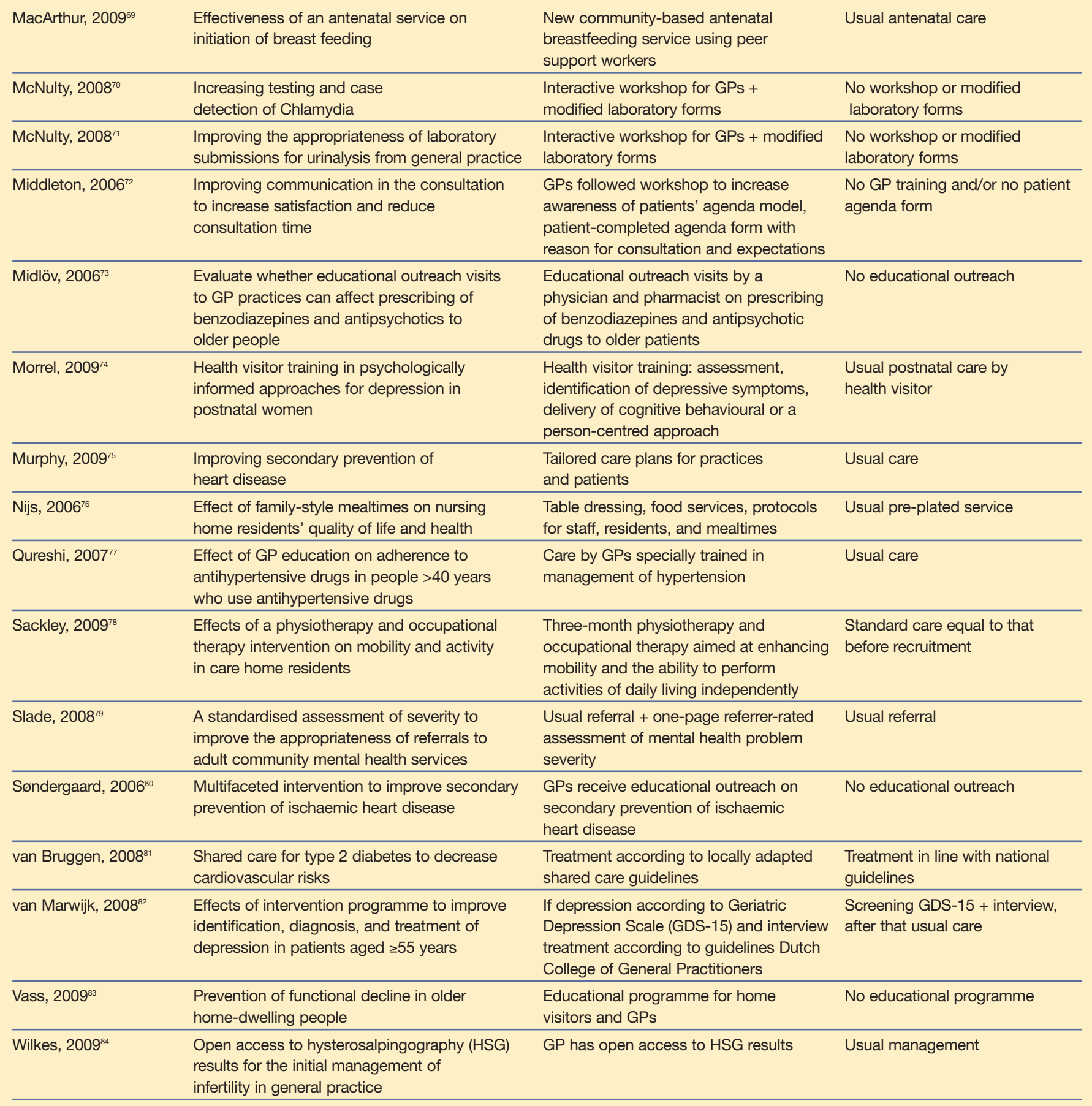

The articles were assessed by two of the authors independently. In case of disagreement, consensus was reached by discussion with a third author. A distinction was made between cluster randomised trials and individually randomised trials, because these study designs have different methodological problems. First, the study assessed how researchers applied the concept of usual care to the control group. For this purpose, an inventory was made of the descriptions that were given of the care in the intervention group and the care in the control group. Second, the risk of behavioural change was assessed according to the following criteria:

- Influences on control caregivers: could the behaviour of the control caregivers possibly be influenced by the researchers? The study assessed whether (a) caregivers were informed 
Table 4. Evaluation of the cluster randomised trials $(n=35)$.

\begin{tabular}{|c|c|c|c|c|c|c|c|c|c|c|c|}
\hline \multirow[b]{2}{*}{ Study } & \multicolumn{5}{|c|}{ Influences on control caregivers } & \multicolumn{6}{|c|}{ Influences on control patients } \\
\hline & $\begin{array}{l}\text { Informed } \\
\text { about } \\
\text { allocation }\end{array}$ & $\begin{array}{l}\text { Informed C } \\
\text { about } \\
\text { content of } \\
\text { intervention }\end{array}$ & $\begin{array}{l}\text { Questionnaire } \\
\text { before or } \\
\text { during } \\
\text { trial }\end{array}$ & $\begin{array}{c}\text { Extra } \\
\text { information/ } \\
\text { training the } \\
\text { on subject }\end{array}$ & $\begin{array}{l}\text { Risk of } \\
\text { learning } \\
\text { effect by } \\
\text { treating } \\
\text { intervention } \\
\text { patients }\end{array}$ & $\begin{array}{l}\text { Informed } \\
\text { consent } \\
\text { given }\end{array}$ & $\begin{array}{l}\text { Informed } \\
\text { about } \\
\text { content of } \\
\text { intervention }\end{array}$ & $\begin{array}{l}\text { Provided } \\
\text { with } \\
\text { extra } \\
\text { information }\end{array}$ & $\begin{array}{l}\text { Informed } \\
\text { about } \\
\text { allocation }\end{array}$ & $\begin{array}{l}\text { Questionnaires/ } \\
\text { examinations } \\
\text { before or } \\
\text { during } \\
\text { intervention }\end{array}$ & $\begin{array}{c}\text { Risk of } \\
\text { contact } \\
\text { with } \\
\text { intervention } \\
\text { patients }\end{array}$ \\
\hline Bebb $2007^{50}$ & $?$ & $?$ & + & - & - & + & $?$ & - & $?$ & + & - \\
\hline Bellon 2008 & + & $?$ & - & $?$ & - & + & - & - & - & - & - \\
\hline Cals $2009^{52}$ & + & $?$ & - & $?$ & - & + & $?$ & $?$ & $?$ & + & - \\
\hline Cullen $2006^{53}$ & + & $?$ & - & - & - & + & $?$ & - & $?$ & - & - \\
\hline Davies 2008 ${ }^{54}$ & $?$ & $?$ & - & + & - & + & $?$ & $?$ & $?$ & + & - \\
\hline de Groot $2007^{55}$ & $?$ & $?$ & - & - & - & + & $?$ & $?$ & + & + & - \\
\hline Downs $2006^{56}$ & $?$ & $?$ & - & - & - & - & - & - & - & - & - \\
\hline Fitzmaurice $2007^{57}$ & $?$ & $?$ & - & - & - & + & $?$ & $?$ & $?$ & - & - \\
\hline Francis $2009^{58}$ & + & $?$ & - & $?$ & - & + & $?$ & $?$ & + & + & $?$ \\
\hline Harmsen $2005^{59}$ & + & $?$ & + & - & - & + & $?$ & - & - & - & - \\
\hline Hayward $2006^{60}$ & $?$ & $?$ & - & - & - & - & - & - & - & - & - \\
\hline Hoddinott 200961 & + & + & - & $?$ & - & + & $?$ & $?$ & $?$ & + & $?$ \\
\hline Hogg $2008^{62}$ & $?$ & $?$ & - & - & - & - & - & - & - & - & - \\
\hline Janssen $2009^{63}$ & + & $?$ & - & + & - & + & $?$ & - & - & + & - \\
\hline Jellema $2005^{64}$ & + & $?$ & - & - & - & + & - & - & - & + & - \\
\hline Kerse $2008^{65}$ & + & $?$ & + & $?$ & - & + & $?$ & $?$ & $?$ & + & - \\
\hline Lester $2007^{66}$ & + & + & - & - & - & + & - & - & - & + & - \\
\hline Lester 200967 & $?$ & $?$ & - & $?$ & - & $?$ & $?$ & $?$ & $?$ & + & - \\
\hline Lo Fo Wong $2006^{68}$ & + & $?$ & + & - & - & + & $?$ & $?$ & - & - & - \\
\hline MacArthur $2009^{69}$ & $?$ & $?$ & - & $?$ & - & - & - & - & - & - & $?$ \\
\hline McNulty $2008^{70}$ & - & - & - & - & - & - & - & - & - & - & - \\
\hline McNulty $2008^{71}$ & - & - & - & - & - & - & - & - & - & - & - \\
\hline Middleton $2006^{72}$ & $?$ & $?$ & - & - & + & + & $?$ & - & - & + & - \\
\hline Midlöv $2005^{73}$ & + & - & - & - & - & - & - & - & - & - & - \\
\hline Morrel 2009 & $?$ & $?$ & - & $?$ & - & + & $?$ & $?$ & $?$ & + & - \\
\hline Murphy $2009^{75}$ & + & $?$ & - & - & - & + & $?$ & $?$ & $?$ & + & - \\
\hline Nijs 2006 & + & $?$ & - & - & - & + & $?$ & $?$ & $?$ & + & - \\
\hline Qureshi $2007^{77}$ & $?$ & $?$ & - & $?$ & - & + & $?$ & $?$ & - & + & - \\
\hline Sackley $2009^{78}$ & $?$ & $?$ & - & $?$ & - & + & $?$ & $?$ & $?$ & + & - \\
\hline Slade $2008^{79}$ & + & $?$ & - & - & - & - & - & - & - & - & - \\
\hline Søndergaard $2005^{80}$ & $?$ & $?$ & + & - & - & - & - & - & - & - & - \\
\hline van Bruggen $2008^{81}$ & + & $?$ & - & - & - & + & $?$ & $?$ & $?$ & + & - \\
\hline van Marwijk $2008^{82}$ & + & $?$ & - & $?$ & - & + & $?$ & $?$ & $?$ & + & - \\
\hline Vass 200983 & + & $?$ & - & - & - & + & $?$ & $?$ & $?$ & + & - \\
\hline Wilkes $2009^{84}$ & + & $?$ & - & + & - & $?$ & $?$ & $?$ & $?$ & - & - \\
\hline
\end{tabular}

$+=$ risk present, - = risk not present, ? = not described in the article.

about the allocation of the patients, (b) caregivers were informed about the content of the intervention, (c) caregivers had to complete questionnaires, (d) caregivers received extra information (for example, guidelines) or training on the subject of the trial, and (e) a learning effect was possible because of contact with patients in the intervention group.

- Influences on control patients: could the behaviour of the patients possibly be influenced by the researchers? The study evaluated whether control patients (a) gave informed consent, (b) were informed about the content of the intervention, (c) were provided with extra information about their condition, (d) knew their allocation status, (e) had to complete questionnaires or undergo examinations, and $(f)$ could have contact with patients in the intervention group. 
Differences between individually randomised and cluster randomised trials were evaluated with a $\chi^{2}$ test.

\section{RESULTS}

A total of 73 articles were identified that met the selection criteria for this overview. Figure 1 presents a flow chart of the search and its results. Overall, 38 individually randomised trials (Tables 1 and 2) ${ }^{12-49}$ and 35 cluster randomised trials (Tables 3 and 4) ) $^{50-84}$ were found. Table 1 shows that the term 'usual care' was used in 33 of the 73 articles. Other expressions were also used, such as 'routine care', ${ }^{15,29,35,63}$ 'usual practice', ${ }^{41}$ 'normal care', ${ }^{34}$ 'standard care', 49,78 'care as usual', ${ }^{55}$ 'usual policy', ${ }^{60}$ 'usual service', ${ }^{76}$ and 'usual management'. ${ }^{84}$ Other authors did not use a specific expression.

\section{Descriptions of usual care by researchers}

In two individually randomised trials, the content of the care in the control group was prescribed by the researchers. Control caregivers were instructed to provide standard asthma care by 6-monthly checkups via a dedicated asthma appointment with a diploma-level asthma nurse, ${ }^{23}$ or perform a 3-monthly measurement of glycosylated haemoglobin $\left(\mathrm{HbA}_{1 \mathrm{c}}\right) .^{19}$ In the remaining 71 trials, control caregivers were allowed to provide care according to their own insight, with some researchers even explicitly asking the caregivers not to change their usual practice.

In four articles it was reported that patients were included in the study after preliminary examinations. Caregivers and patients were informed about the results of these pre-trial investigations, regardless of allocation to the intervention group or control group. ${ }^{25,28,41,82}$

\section{Influences on control caregivers}

Half of all trials described that control caregivers were informed about the allocation status of their patients or practice. About half of the authors made no mention of caregivers' awareness of the allocation status. The proportions were similar for individually and cluster randomised trials (Table 5).

In 16 individually randomised trials, caregivers provided the intervention as well as usual care, indicating that they were aware of both arms of the study. In 22 of the individually randomised trials, the information given to caregivers was not described in the article. In two trials it was reported that caregivers were not informed about the content of the intervention. This was possible because caregivers had no involvement in the development and distribution of the intervention (an educational booklet), ${ }^{16}$ or were blinded to the treatment protocol, which was performed by a separate team. ${ }^{40}$

In the majority of articles on cluster randomised trials, the information provided to control caregivers was not described. Only three papers explicitly stated that control caregivers were not aware of the exact content of the intervention; they received either an unrelated educational module,,$^{70,71}$ or neutral information about the trial without an explanation of the intervention. ${ }^{73}$

In some individually randomised and some cluster randomised trials, control caregivers were asked to complete questionnaires before or during the intervention period. In individually randomised trials, control caregivers recorded patient complaints, ${ }^{12,25}$ or evaluated the consultation. ${ }^{14}$ In cluster randomised trials, caregivers were asked about the care they provided before the start of the trial, ${ }^{50,65}$ were asked to evaluate the consultation, ${ }^{21,68}$ or were asked to record patient complaints. ${ }^{80}$

Understandably, control caregivers in the individually randomised trials were more often given extra information on the subject under study compared to caregivers in the cluster randomised trials. In 11 individually randomised trials, caregivers of control patients received training because they also had to provide the intervention to patients allocated to the intervention group. In one cluster randomised trial, all caregivers, including the usual care group, received the national guidelines on the subject under study, ${ }^{54}$ and in two trials they were invited to an initiation symposium. ${ }^{63,84}$ In another trial, the risk of behavioural change among caregivers was reduced by excluding those who were involved in the development of study guidelines. ${ }^{53}$

The possibility of a learning effect of caregivers was more often seen in individually randomised trials than in cluster randomised trials. This problem was more evident in individually randomised trials because the caregiver who provided the intervention to the intervention group also often continued to provide (usual) care to control patients. Less than half of individually randomised trials and one cluster randomised trial showed a risk of a learning effect in caregivers. In cluster randomised trials, caregivers might be influenced by information about the intervention communicated via patients who had this information. ${ }^{72}$

\section{Influences on control patients}

In nine of the cluster randomised trials, it was not necessary to inform control patients about the trial because the study data were gathered from anonymous electronic patient records and therefore consent was not considered necessary. In these latter trials, no risks of behavioural change in control patients existed. In almost all the individually randomised trials it was described that informed consent was obtained from control patients (Table 5). 
Table 5. Comparison of influences on usual care control group between individually randomised trials and cluster randomised trials.

\begin{tabular}{|c|c|c|c|c|c|c|c|}
\hline & \multicolumn{6}{|c|}{ Possible influence on usual care control group } & \multirow[b]{3}{*}{$P$-value } \\
\hline & \multicolumn{3}{|c|}{ Individually randomised trials $(n=38)$} & \multicolumn{3}{|c|}{ Cluster randomised trials $(n=35)$} & \\
\hline & Yes & No & Not described & Yes & No & Not described & \\
\hline \multicolumn{8}{|l|}{ Caregivers } \\
\hline $\begin{array}{l}\text { Informed about allocation } \\
\text { of patients }\end{array}$ & 15 & 8 & 15 & 19 & 2 & 14 & 0.140 \\
\hline $\begin{array}{l}\text { Informed about content } \\
\text { of intervention }\end{array}$ & 16 & 2 & 22 & 2 & 3 & 30 & 0.002 \\
\hline $\begin{array}{l}\text { Questionnaires before or } \\
\text { during trial }\end{array}$ & 3 & 35 & 0 & 5 & 28 & 0 & 0.280 \\
\hline Extra information or training & 11 & 14 & 13 & 3 & 21 & 11 & 0.049 \\
\hline $\begin{array}{l}\text { Risk of learning effect by } \\
\text { treating intervention patients }\end{array}$ & 15 & 18 & 5 & 1 & 34 & 0 & $<0.001$ \\
\hline \multicolumn{8}{|l|}{ Patients } \\
\hline Informed consent given & 35 & 2 & 1 & 24 & 9 & 2 & 0.036 \\
\hline $\begin{array}{l}\text { Informed about content } \\
\text { of intervention }\end{array}$ & 3 & 4 & 31 & 0 & 12 & 23 & 0.018 \\
\hline Provided with extra information & 8 & 23 & 7 & 0 & 17 & 18 & 0.001 \\
\hline Informed about allocation & 22 & 2 & 14 & 2 & 17 & 16 & $<0.001$ \\
\hline Questionnaires or examinations & 34 & 4 & 0 & 20 & 15 & 0 & 0.002 \\
\hline $\begin{array}{l}\text { Risk of contact with } \\
\text { intervention patients }\end{array}$ & 2 & 22 & 4 & 0 & 32 & 3 & 0.200 \\
\hline
\end{tabular}

In most trials, the article did not describe whether (or not) any information was provided to control patients. Therefore, it was not possible to judge these trials with respect to what influence study information may have had on control patients. If information had been given to control patients, most researchers did not inform patients about the content of the intervention.

In eight individually randomised trials, patients in the control group received information that could have influenced their behaviour. They were offered an information sheet, ${ }^{33,36,49}$ received extra treatment advice, ${ }^{19,47}$ or underwent a medical investigation before entering the trial. ${ }^{25,28,41}$ This problem was not found in cluster randomised trials.

Patients were informed about their allocation in more than half of individually randomised trials. This was the case in only two of the cluster randomised trials. Unfortunately, in $25 \%$ of the papers the authors did not mention whether patients knew about their allocation.

In most individually randomised trials, control patients were asked to complete questionnaires or to undergo examinations. About half of the cluster randomised trials gathered their information from electronic patient records or after the intervention period. ${ }^{59}$

The risk of influencing behaviour through contact between control patients and intervention patients was not evident. It was, however, difficult to properly assess this risk from the limited descriptions provided by the researchers. In two trials the authors acknowledged that between-group contact could have taken place..$^{24,37}$ They described the potential problem and stated that they had no reason to believe that chance contact between the intervention group and the control patients had led to behavioural changes.

\section{DISCUSSION}

In this overview, different interpretations of the concept of usual care were observed across the studies. Factors in the design of trials with a usual care control group influencing behaviour of control caregivers and participants (such as information about allocation or intervention) were present not only in individually randomised trials but also in cluster randomised trials.

\section{Description of usual care by researchers}

This overview shows that the usual care concept is interpreted differently across the research community. According to some authors, one specific, predefined treatment should be chosen and be given to all subjects in the control group..$^{11,85}$ This point of view argues that variation in treatments for control patients makes trial results difficult to interpret and generalise. In contrast, others try to improve external validity by advising that patients in a usual care control group should be confronted with the heterogeneity of treatments available in real, daily practice, rather than receiving a treatment chosen by the researchers. ${ }^{86,87}$ This view corresponds with the conclusion of two meetings of the National Institute of Mental Health (US) 
where usual care was defined as the wide range of care that is provided in a community whether it is adequate or not, without a normative judgment' ${ }^{88,89}$ In these meetings it was agreed that trials should have a usual care control group when they aim to prove superiority of a new intervention or approach over usual care in the community.

Thus, in the ideal situation, the usual care control group is not influenced at all. However, the ethical requirements of research and the proper conduct of trials can disturb the naturalistic character of a usual care approach.

\section{Behavioural change in a usual care control group}

This overview shows that risk of (unwanted) behavioural changes among caregivers and patients in a usual care control group is often present. Cluster randomisation is often seen as the solution for this problem. However, this study found that control caregivers' awareness of study conditions could influence their behaviour even in cluster randomised trials. Thus, in designing a study, researchers should ask themselves if caregivers need to be informed about the allocation of their patients or practice and about the content of the intervention. They should at least avoid providing information about the intervention to the control caregivers.

Also, when designing a trial, researchers should ask themselves to what extent control patients need to be informed about the trial, about the content of the intervention, and about their allocation. Possible solutions to diminish the risk of study-induced behavioural change include giving patients in the control group neutral information, ${ }^{40,51}$ or not disclosing the presence of the other study arm. The latter solution is called the Zelen design. . $8,90,91$ However, many medical ethical committees refuse to accept this design, because they do not support withholding of information..$^{22}$

Regarding the use of questionnaires and examinations, researchers should ask themselves whether these are needed and, if so, what questions are essential. In some trials, participants were asked to complete extensive baseline questionnaires or think about questions that would otherwise not have crossed their minds. ${ }^{72}$ This may have changed their help-seeking behaviour. Finally, especially in individually randomised trials, researchers should be alert to the possibility of contact between control patients and intervention patients.

\section{Strengths and limitations of the study}

This overview has revealed that authors rarely describe the information given to control caregivers and control patients in their paper, making it difficult for readers to evaluate the adequacy of the design. The fact that not all the required information was actually described in the research papers is a limitation of this overview. Often, it was not possible to fully appreciate the attempts of the researchers to ensure usual care was maintained in the control group. Because of this scarce information, it was often impossible to establish with certainty to what extent the observed problems actually influenced the final study results. It has been reported that results are difficult to interpret without knowing the nature of usual care. ${ }^{8,9}$ This study has provided an overview of the risks of behavioural change, but without the intention to be exhaustive. However, it has given an impression of the key issues affecting usual care control groups in this type of trial.

This overview addresses an overlooked issue in the medical literature, namely the design of a usual care control group in pragmatic trials. Because the term 'usual care' is not used consistently by researchers, trials could not easily be identified by a search in electronic databases. For this reason, the researchers manually searched three journals that regularly publish pragmatic trials in primary care with a usual care control group. In this way it was possible to provide an adequate impression of the current issues.

\section{Recommendations for future research}

In conclusion, researchers should carefully consider the design of a usual care control group, and consider ways to diminish the risk of study-induced behavioural change of caregivers and patients. As cluster randomised trials seem to be less sensitive to this problem, it may be advisable to consider this type of study design in preference to individually randomised trials. However, even when using cluster randomisation, researchers should bear in mind the risks of behavioural change in the usual care control group. In addition, this overview supports the argument that researchers should provide a better description of the design of the control group and the care provided to them. Many researchers do not report which, if any, information has been given to control caregivers and control patients, making it difficult to evaluate the adequacy of the study design in terms of internal and external validity. In the CONSORT statement extension for pragmatic trials, it is recommended to describe the control group in as much detail as the intervention group. The authors of the present study recommend making this recommendation more specific (Table 6). This implies that, in addition to a description of the intervention group, the reader requires a detailed description of the instructions given to control caregivers, as well as the information given to control patients that are 
Table 6. The CONSORT statement for randomised controlled trials, ${ }^{93}$ with proposed adaptations in italics.

\begin{tabular}{|c|c|c|}
\hline & $\begin{array}{l}\text { Item } \\
\text { number }\end{array}$ & Descriptor \\
\hline Title and abstract & 1 & How participants were allocated to interventions (for example, 'random allocation', 'randomised', or 'randomly assigned'). \\
\hline \multicolumn{3}{|l|}{ Introduction } \\
\hline Background & 2 & Scientific background and explanation of rationale. \\
\hline \multicolumn{3}{|l|}{ Methods } \\
\hline Participants & 3 & $\begin{array}{l}\text { Eligibility criteria for participants and the settings and locations where the data were collected, specifying the } \\
\text { information provided to participants. }\end{array}$ \\
\hline Interventions & 4 & $\begin{array}{l}\text { Precise details of the interventions intended for each group and how and when they were actually administered, } \\
\text { who provided the intervention, and what information caregivers received. }\end{array}$ \\
\hline Control group & & $\begin{array}{l}\text { Clear description of the care in the control group, details about the information provided and instructions given } \\
\text { to control caregivers, including the rationale for blinding or not blinding caregivers to allocation status, details } \\
\text { about the information provided, and instructions given to control patients, including the rationale for blinding or } \\
\text { not blinding patients to allocation status. }\end{array}$ \\
\hline Objectives & 5 & Specific objectives and hypotheses. \\
\hline Outcomes & 6 & $\begin{array}{l}\text { Clearly defined primary and secondary outcome measures and, when applicable, any methods used to enhance } \\
\text { the quality of measurements (for example, multiple observations, training of assessors). }\end{array}$ \\
\hline Sample size & 7 & How sample size was determined and, when applicable, explanation of any interim analyses and stopping rules. \\
\hline \multicolumn{3}{|l|}{ Randomisation } \\
\hline Sequence generation & 8 & $\begin{array}{l}\text { Method used to generate the random allocation sequence, including details of any restriction (for example, blocking, } \\
\text { stratification). }\end{array}$ \\
\hline Allocation concealment & 9 & $\begin{array}{l}\text { Method used to implement the random allocation sequence (for example, numbered containers or central telephone), } \\
\text { clarifying whether the sequence was concealed until interventions were assigned. }\end{array}$ \\
\hline Implementation & 10 & Who generated the allocation sequence, who enrolled participants, and who assigned participants to their groups. \\
\hline Blinding (masking) & 11 & $\begin{array}{l}\text { Whether participants, those administering the interventions, those providing care in the control group, and those } \\
\text { assessing the outcomes were blinded to group assignment. If done, how the success of blinding was evaluated. }\end{array}$ \\
\hline Statistical methods & 12 & $\begin{array}{l}\text { Statistical methods used to compare groups for primary outcome(s); methods for additional analyses, such as } \\
\text { subgroup analyses and adjusted analyses. }\end{array}$ \\
\hline \multicolumn{3}{|l|}{ Results } \\
\hline Participants' flow & 13 & $\begin{array}{l}\text { Flow of individual participants through each stage (a diagram is strongly recommended). Specifically, for each } \\
\text { group report the numbers of participants randomly assigned, receiving intended treatment, completing the study } \\
\text { protocol, and analysed for the primary outcome. Describe protocol deviations from study as planned, together with } \\
\text { reasons. }\end{array}$ \\
\hline Recruitment & 14 & Dates defining the periods of recruitment and follow-up. \\
\hline Baseline data & 15 & Baseline information for each group. \\
\hline Numbers analysed & 16 & $\begin{array}{l}\text { Number of participants (denominator) in each group included in each analysis and whether the analysis was by } \\
\text { intention to treat. State the results in absolute numbers when feasible (for example, } 10 / 20 \text { not } 50 \% \text { ). }\end{array}$ \\
\hline Outcomes and estimation & 17 & $\begin{array}{l}\text { For each primary and secondary outcome, a summary of results for each group, and the estimated effect size and } \\
\text { its precision (for example, } 95 \% \text { confidence interval). }\end{array}$ \\
\hline Ancillary analyses & 18 & $\begin{array}{l}\text { Address multiplicity by reporting any other analyses performed, including subgroup analyses and adjusted } \\
\text { analyses, indicating those pre-specified and those exploratory. }\end{array}$ \\
\hline Adverse events & 19 & All important adverse events or side effects in each intervention group. \\
\hline \multicolumn{3}{|l|}{ Discussion } \\
\hline Interpretation & 20 & $\begin{array}{l}\text { Interpretation of the results, taking into account study hypotheses, sources of potential bias or imprecision, and } \\
\text { the dangers associated with multiplicity of analyses and outcomes. }\end{array}$ \\
\hline Generalisability & 21 & Generalisability (external validity) of the trial findings. \\
\hline Overall evidence & 22 & General interpretation of the results in the context of current evidence. \\
\hline
\end{tabular}

supposed to receive usual care. This will allow the reader to evaluate whether the care provided to the control group is sufficiently representative for the usual care in daily practice.

\section{Competing interests}

The authors have stated that there are none.

\section{Discuss this article}

Contribute and read comments about this article on the

Discussion Forum: http://www.rcgp.org.uk/bjgp-discuss

\section{REFERENCES}

1. Roland M, Torgerson DJ. What are pragmatic trials? BMJ 1998; 316(7127): 285. 
2. Barkauskas VH, Lusk SL, Eakin BL. Selecting control interventions for clinical outcome studies. West J Nurs Res 2005; 27(3): 346-363.

3. Campbell MK, Elbourne DR, Altman DG. CONSORT statement: extension to cluster randomised trials. BMJ 2004; 328(7441): 702-708.

4. Moher D, Schulz KF, Altman D. The CONSORT Statement: revised recommendations for improving the quality of reports of parallelgroup randomized trials 2001. Explore (NY) 2005; 1(1): 40-45.

5. Zwarenstein $\mathrm{M}$, Treweek S, Gagnier JJ, et al. Improving the reporting of pragmatic trials: an extension of the CONSORT statement. BMJ 2008; 337: a2390.

6. Eldridge S, Ashby D, Bennett C, et al. Internal and external validity of cluster randomised trials: systematic review of recent trials. BMJ 2008; 336(7649): 876-880.

7. Puffer S, Torgerson D, Watson J. Evidence for risk of bias in cluster randomised trials: review of recent trials published in three general medical journals. BMJ 2003; 327(7418): 785-789.

8. Mant D. The problem with usual care. Br J Gen Pract 2008; 58(556): 755-756.

9. Somerville S, Hay E, Lewis M, et al. Content and outcome of usual primary care for back pain: a systematic review. Br J Gen Pract 2008; 58(556): 790-797, i-vi.

10. Gartlehner G, Hansen RA, Nissman D, et al. A simple and valid tool distinguished efficacy from effectiveness studies. J Clin Epidemiol 2006; 59(10): 1040-1048.

11. Godwin M, Ruhland L, Casson I, et al. Pragmatic controlled clinical trials in primary care: the struggle between external and internal validity. BMC Med Res Methodol 2003; 3: 28

12. Boivin JM, Poupon-Lemarquis L, Iraqi W, et al. A multifactorial strategy of pain management is associated with less pain in scheduled vaccination of children. A study realized by family practitioners in 239 children aged 4-12 years old. Fam Pract 2008; 25(6): 423-429.

13. Community Pharmacy Medicines Management Project Evaluation Team. The MEDMAN study: a randomized controlled trial of community pharmacy-led medicines management for patients with coronary heart disease. Fam Pract 2007; 24(2): 189-200.

14. Christensen KS, Toft T, Frostholm L, et al. Screening for common mental disorders: who will benefit? Results from a randomised clinical trial. Fam Pract 2005; 22(4): 428-434.

15. Coppin R, Wicke D, Little P. Managing earwax in primary care: efficacy of self-treatment using a bulb syringe. Br J Gen Pract 2008; 58(546): 44-49.

16. Crilly M, Esmail A. Randomised controlled trial of a hypothyroid educational booklet to improve thyroxine adherence. $\mathrm{Br} J$ Gen Pract 2005; 55(514): 362-368.

17. Daley A, Winter $\mathrm{H}$, Grimmett C, et al. Feasibility of an exercise intervention for women with postnatal depression: a pilot randomised controlled trial. Br J Gen Pract 2008; 58(548): 178-183.

18. Dennis CL, Hodnett E, Kenton L, et al. Effect of peer support on prevention of postnatal depression among high risk women: multisite randomised controlled trial. BMJ 2009; 338: a3064.

19. Farmer A, Wade A, Goyder E, et al. Impact of self monitoring of blood glucose in the management of patients with non-insulin treated diabetes: open parallel group randomised trial. BMJ 2007; 335(7611): 132

20. Gorgels WJ, Oude Voshaar RC, et al. Consequences of a benzodiazepine discontinuation programme in family practice on psychotropic medication prescription to the participants. Fam Pract 2007; 24(5): $504-510$

21. Green J, Denham A, Ingram J, et al. Treatment of menopausal symptoms by qualified herbal practitioners: a prospective, randomized controlled trial. Fam Pract 2007; 24(5): 468-474.

22. Griffiths C, Motlib J, Azad A, et al. Randomised controlled trial of a layled self-management programme for Bangladeshi patients with chronic disease. Br J Gen Pract 2005; 55(520): 831-837.

23. Gruffydd-Jones K, Hollinghurst S, Ward S, Taylor G. Targeted routine asthma care in general practice using telephone triage. Br J Gen Pract 2005; 55(521): 918-923.

24. Hamilton W, Russell D, Stabb C, et al. The effect of patient selfcompletion agenda forms on prescribing and adherence in general practice: a randomized controlled trial. Fam Pract 2007; 24(1): 77-83.

25. Hoefman E, van Weert HC, Reitsma JB, et al. Diagnostic yield of patient-activated loop recorders for detecting heart rhythm abnormalities in general practice: a randomised clinical trial. Fam Pract 2005; 22(5): 478-484

26. Holland R, Brooksby I, Lenaghan E, et al. Effectiveness of visits from community pharmacists for patients with heart failure: HeartMed randomised controlled trial BMJ 2007:334(7603): 1098.

27. Holland R, Lenaghan E, Harvey I, et al. Does home based medication review keep older people out of hospital? The HOMER randomised controlled trial. BMJ 2005; 330(7486): 293.

28. Hunkeler EM, Katon W, Tang L, et al. Long term outcomes from the IMPACT randomised trial for depressed elderly patients in primary care. BMJ 2006; 332(7536): 259-263.

29. Khunti K, Stone MA, Burden AC, et al. Randomised controlled trial of near-patient testing for glycated haemoglobin in people with type 2 diabetes mellitus. Br J Gen Pract 2006; 56(528): 511-517.

30. Lawton BA, Rose SB, Raina EC, et al. Exercise on prescription for women aged 40-74 recruited through primary care: two year randomised controlled trial. Br J Sports Med 2009; 43(2): 120-123.

31. Leong KC, Chen WS, Leong KW, et al. The use of text messaging to improve attendance in primary care: a randomized controlled trial. Fam Pract 2006; 23(6): 699-705.

32. Liew SM, Tong SF, Lee VK, et al. Text messaging reminders to reduce non-attendance in chronic disease follow-up: a clinical trial. $\mathrm{Br} J \mathrm{Gen}$ Pract 2009; 59: 916-920

33. Linschoten LR, van MM, Berger MY, et al. Supervised exercise therapy versus usual care for patellofemoral pain syndrome: an open label randomised controlled trial. BMJ 2009; 339: b4074.

34. Little P, Lewith G, Webley F, et al. Randomised controlled trial of Alexander technique lessons, exercise, and massage (ATEAM) for chronic and recurrent back pain. BMJ 2008; 337: a884.

35. Martins N, Morris P, Kelly PM. Food incentives to improve completion of tuberculosis treatment: randomised controlled trial in Dili, TimorLeste. BMJ 2009; 339: b4248.

36. McMahon L, Foran KM, Forrest SD, et al. Graduate mental health worker case management of depression in UK primary care: a pilot study. Br J Gen Pract 2007; 57(544): 880-885.

37. McManus RJ, Mant J, Roalfe A, et al. Targets and self monitoring in hypertension: randomised controlled trial and cost effectiveness analysis. BMI 2005; 331(7515): 493.

38. Muirhead PE, Butcher G, Rankin J, Munley A. The effect of a programme of organised and supervised peer support on the initiation and duration of breastfeeding: a randomised trial. Br J Gen Pract 2006; 56(524): 191-197.

39. Nanchahal K, Townsend J, Letley L, et al. Weight-management interventions in primary care: a pilot randomised controlled trial. $\mathrm{Br} J$ Gen Pract 2009; 59(562): e157-el66.

40. Norg RJ, van de BK, Portegijs PJ, et al. The effectiveness of a treatment protocol for male lower urinary tract symptoms in general practice: a practical randomised controlled trial. Br J Gen Pract 2006; 56(533): 938-944

41. Roberts L, Wilson S, Singh S, et al. Gut-directed hypnotherapy for irritable bowel syndrome: piloting a primary care-based randomised controlled trial. Br J Gen Pract 2006; 56(523): 115-121.

42. Schreuders B, van Oppen P, van Marwijk HW, et al. Frequent attenders in general practice: problem solving treatment provided by nurses. BMC Fam Pract 2005; 6: 42

43. Schroeder K, Fahey T, Hollinghurst S, Peters TJ. Nurse-led adherence support in hypertension: a randomized controlled trial. Fam Pract 2005 22(2): 144-151

44. Thomas KJ, MacPherson H, Thorpe L, et al. Randomised controlled trial of a short course of traditional acupuncture compared with usual care for persistent non-specific low back pain. BMJ 2006; 333(7569): 623.

45. Thomsen JL, Parner ET, Karlsmose B, et al. Effect of preventive health screening on long-term primary health care utilization. A randomized controlled trial. Fam Pract 2005; 22(3): 242-248.

46. Van Rijn RM, van Os AG, Kleinrensink GJ, et al. Supervised exercises for adults with acute lateral ankle sprain: a randomised controlled trial. Br J Gen Pract 2007; 57(543): 793-800.

47. Vicens C, Fiol F, Llobera J, et al. Withdrawal from long-term benzodiazepine use: randomised trial in family practice. $\mathrm{Br} J \mathrm{Gen}$ Pract 2006; 56(533): 958-963.

48. Wake M, Baur LA, Gerner B, et al. Outcomes and costs of primary care surveillance and intervention for overweight or obese children: the LEAP 2 randomised controlled trial. BMJ 2009; 339: b3308.

49. Williams KS, Assassa RP, Cooper NJ, et al. Clinical and cost-effectiveness of a new nurse-led continence service: a randomised controlled trial. $\mathrm{Br}$ J Gen Pract 2005; 55(518): 696-703.

50. Bebb C, Kendrick D, Coupland C, et al. A cluster randomised controlled 
trial of the effect of a treatment algorithm for hypertension in patients with type 2 diabetes. Br J Gen Pract 2007; 57(535): 136-143.

51. Bellon JA, Rodriguez-Bayon A, de Dios LJ, Torres-Gonzalez F. Successfu GP intervention with frequent attenders in primary care: randomised controlled trial. Br J Gen Pract 2008; 58(550): 324-330.

52. Cals JW, Butler CC, Hopstaken RM, et al. Effect of point of care testing for $\mathrm{C}$ reactive protein and training in communication skills on antibiotic use in lower respiratory tract infections: cluster randomised trial. BMJ 2009; 338: b1374.

53. Cullen W, Stanley J, Langton D, et al. Hepatitis C infection among injecting drug users in general practice: a cluster randomised controlled trial of clinical guidelines' implementation. Br J Gen Pract 2006; 56(532): 848-856.

54. Davies MJ, Heller S, Skinner TC, et al. Effectiveness of the diabetes education and self management for ongoing and newly diagnosed (DESMOND) programme for people with newly diagnosed type 2 diabetes: cluster randomised controlled trial. BMJ 2008; 336(7642): 491-495.

55. De Groot M, de Keijser J, Neeleman J, et al. Cognitive behaviour therapy to prevent complicated grief among relatives and spouses bereaved by suicide: cluster randomised controlled trial. BMJ 2007; 334(7601): 994.

56. Downs M, Turner S, Bryans M, et al. Effectiveness of educational interventions in improving detection and management of dementia in primary care: cluster randomised controlled study. BMJ 2006; 332(7543): 692-696

57. Fitzmaurice DA, Hobbs FD, Jowett S, et al. Screening versus routine practice in detection of atrial fibrillation in patients aged 65 or over: cluster randomised controlled trial. BMJ 2007; 335(7616): 383 .

58. Francis NA, Butler CC, Hood K, et al. Effect of using an interactive booklet about childhood respiratory tract infections in primary care consultations on reconsulting and antibiotic prescribing: a cluster randomised controlled trial. BMJ 2009; 339: b2885.

59. Harmsen H, Bernsen R, Meeuwesen L, et al. The effect of educational intervention on intercultural communication: results of a randomised controlled trial. Br J Gen Pract 2005; 55(514): 343-350.

60. Hayward AC, Harling R, Wetten S, et al. Effectiveness of an influenza vaccine programme for care home staff to prevent death, morbidity, and health service use among residents: cluster randomised controlled trial. BMJ 2006; 333(7581): 1241.

61. Hoddinott P, Britten J, Prescott GJ, et al. Effectiveness of policy to provide breastfeeding groups (BIG) for pregnant and breastfeeding mothers in primary care: cluster randomised controlled trial. BMJ 2009, 338: a3026.

62. Hogg W, Lemelin J, Graham ID, et al. Improving prevention in primary care: evaluating the effectiveness of outreach facilitation. Fam Pract 2008; 25(1): 40-48.

63. Janssen PG, Gorter KJ, Stolk RP, Rutten GE. Randomised controlled trial of intensive multifactorial treatment for cardiovascular risk in patients with screen-detected type 2 diabetes: 1-year data from the ADDITION Netherlands study. Br J Gen Pract 2009; 59(558): 43-48.

64. Jellema P, van der Windt DA, van der Horst HE, et al. Should treatmen of (sub)acute low back pain be aimed at psychosocial prognostic factors? Cluster randomised clinical trial in general practice. BMJ 2005; 331(7508): 84.

65. Kerse N, Peri K, Robinson E, et al. Does a functional activity programme improve function, quality of life, and falls for residents in long term care? Cluster randomised controlled trial. BMJ 2008; 337: a1445.

66. Lester H, Freemantle N, Wilson S, et al. Cluster randomised controlled trial of the effectiveness of primary care mental health workers. Br J Gen Pract 2007; 57(536): 196-203.

67. Lester H, Birchwood M, Freemantle N, et al. REDIRECT: cluster randomised controlled trial of GP training in first-episode psychosis. $\mathrm{Br}$ J Gen Pract 2009; 59(563): e183-e190.

68. Lo Fo WS, Wester F, Mol SS, Lagro-Janssen TL. Increased awareness of intimate partner abuse after training: a randomised controlled trial. $\mathrm{Br}$ Gen Pract 2006; 56(525): 249-257.

69. MacArthur C, Jolly K, Ingram L, et al. Antenatal peer support workers and initiation of breast feeding: cluster randomised controlled trial. BMJ 2009; 338: b131.

70. McNulty CA, Thomas M, Bowen J, et al. Interactive workshops increase chlamydia testing in primary care - a controlled study. Fam Pract 2008; 25(4): 279-286.

71. McNulty CA, Thomas M, Bowen J, et al. Improving the appropriatenes of laboratory submissions for urinalysis from general practice. Fam Pract 2008; 25(4): 272-278.
72. Middleton JF, McKinley RK, Gillies CL. Effect of patient completed agenda forms and doctors' education about the agenda on the outcome of consultations: randomised controlled trial. BMJ 2006; 332(7552): 1238-1242.

73. Midlöv P, Bondesson A, Eriksson T, et al. Effects of educationa outreach visits on prescribing of benzodiazepines and antipsychotic drugs to elderly patients in primary health care in southern Sweden. Fam Pract 2006; 23(1): 60-64.

74. Morrell CJ, Slade P, Warner R, et al. Clinical effectiveness of health visitor training in psychologically informed approaches for depression in postnatal women: pragmatic cluster randomised trial in primary care. BMJ 2009; 338: a3045.

75. Murphy AW, Cupples ME, Smith SM, et al. Effect of tailored practice and patient care plans on secondary prevention of heart disease in general practice: cluster randomised controlled trial. BMJ 2009; 339: b4 4220 .

76. Nijs KA, de Graaf C, Kok FJ, van Staveren WA. Effect of family style mealtimes on quality of life, physical performance, and body weight of nursing home residents: cluster randomised controlled trial. BMJ 2006; 332(7551): 1180-1184.

77. Qureshi NN, Hatcher J, Chaturvedi N, Jafar TH. Effect of general practitioner education on adherence to antihypertensive drugs: cluster randomised controlled trial. BMJ 2007; 335(7628): 1030.

78. Sackley CM, van den Berg ME, Lett K, et al. Effects of a physiotherapy and occupational therapy intervention on mobility and activity in care home residents: a cluster randomised controlled trial. BMJ 2009; 339: b3123.

79. Slade M, Gask L, Leese M, et al. Failure to improve appropriateness of referrals to adult community mental health services - lessons from multi-site cluster randomized controlled trial. Fam Pract 2008; 25(3): $181-190$.

80. Søndergaard J, Hansen DG, Aarslev P, Munck AP. A multifaceted intervention according to the Audit Project Odense method improved secondary prevention of ischemic heart disease: a randomised controlled trial. Fam Pract 2006; 23(2): 198-202.

81. Van Bruggen BR, Gorter KJ, Stolk RP, et al. Implementation of locally adapted guidelines on type 2 diabetes. Fam Pract 2008; 25(6): 430-437.

82. Van Marwijk HW, Ader H, de Haan M, Beekman A. Primary care management of major depression in patients aged $\geq 55$ years: outcome of a randomised clinical trial. Br J Gen Pract 2008; 58(555): 680-6II

83. Vass M, Avlund K, Siersma V, Hendriksen C. A feasible model for prevention of functional decline in older home-dwelling people - the GP role. A municipality-randomized intervention trial. Fam Pract 2009; 26(1): 56-64.

84. Wilkes S, Murdoch A, Steen N, et al. Open Access Tubal aSsessment for the initial management of infertility in general practice (the OATS trial) a pragmatic cluster randomised controlled trial. Br J Gen Pract 2009; 59(562): 329-335.

85. Fransen GA, van Marrewijk CJ, Mujakovic S, et al. Pragmatic trials in primary care. Methodological challenges and solutions demonstrated by the DIAMOND study. BMC Med Res Methodol 2007; 7: 16

86. Degenholtz HB, Parker LS, Reynolds CF. Trial design and informed consent for a clinic-based study with a treatment as usual control arm. Ethics Behav 2002; 12(1): 43-62.

87. Staines GL, McKendrick K, Perlis T , et al. Sequential assignment and treatment-as-usual. Alternatives to standard experimental designs in field studies of treatment efficacy. Eval Rev 1999; 23(1): 47-76.

88. NIH program on Clinical Research Policy Analysis and Coordination. Meeting proceedings. Considering usual medical care in clinical trial design: scientific and ethical issues. Bethesda, MD: 14-15 November 2005.

89. NIH Rockville. Meeting summary. Treatment as usual: measurement, design and ethics. Rockville, MD: 4-5 November 2002.

90. Edwards SJ, Braunholtz DA, Lilford RJ, Stevens AJ. Ethical issues in the design and conduct of cluster randomised controlled trials. BMJ 1999; 318(7195): 1407-1409.

91. Huibers MJ, Bleijenberg G, Beurskens AJ, et al. An alternative tria design to overcome validity and recruitment problems in primary care research. Fam Pract 2004; 21(2): 213-218.

93. Macpherson H. Pragmatic clinical trials. Complement Ther Med 2004; 12(2-3): 136-140.

93. Schellings R, Kessels AG, Ter RG, Kleijnen J, et al. Members of research ethics committees accepted a modification of the randomized consent design. J Clin Epidemiol 2005; 58(6): 589-594. 